\title{
Prevención de la violencia escolar cara a cara $y$ virtual en bachillerato
}

\author{
Rocío Martínez Vilchis*, Jesús Pozas Rivera, Karen Jiménez Arriga, Tania Morales Reynoso, \\ David Aarón Miranda, María Estela Delgado Maya y Verónica Cuenca Sánchez \\ Universidad Autónoma del Estado de México, Facultad de Ciencias de la Conducta
}

(Recibido 16 Mayo, 2015; Aceptado 23 Junio, 2015)

\begin{abstract}
RESUMEN: En el presente artículo se pretende aportar una visión completa de las problemáticas de bullying y cyberbullying en alumnos de bachillerato, así como algunas herramientas que sirven de factor prevención en casos de violencia en entornos cara a cara y en entornos virtuales, como lo son las competencias genéricas y las competencias digitales; todo esto partir de tres estudios, dos transversales descriptivos y uno correlacional, realizados en estudiantes de bachillerato de la Universidad Autónoma del Estado de México. En estos estudios se aplicaron cuestionarios de autoinforme para obtener datos sobre la percepción que tienen los adolescentes sobre estas problemáticas, y en el caso de las competencias genéricas y digitales, el nivel de dominio que tienen de estas.
\end{abstract}

Palabras clave: bullying, cyberbullying, competencias genéricas, competencias digitales.

\section{Prevention of school violence face to face and virtual in the high school}

ABSTRACT: The present article pretends contribute a complete vision about the problems of bullying and cyberbullying in high school students, and some tools who helps as a prevention factor in face to face environments and virtual environments, as generic competences and digital competence; all this starting with three researches, two of these descriptive transversal studies and one correlational study, made on high school students of the Universidad Autónoma del Estado de México; in these studies were applied self-report questionnaires to obtain information about the perception of the teenagers about these issues, and in case of generic competences and digital competence, the knowledge they have. Keywords: bullying, cyberbullying, generic competences, digital competence.

\section{INTRODUCCIÓN}

La violencia escolar es una problemática amplia en la que se incluye la violencia entre iguales (Del Rey y Ortega, 2007) que puede presentarse tanto en la interacción cara a cara como de manera virtual, fenómenos denominados bullying y cyberbullying. Se dice que un alumno es víctima de bullying cuando recibe repetitivamente acciones negativas por parte de otro alumno o grupo de ellos, lo cual manifiesta una relación de poder asimétrica y dificultades del alumno victimizado para defenderse (Olweus, 2006). En relación con lo anterior, se 
considera cyberbullying a los actos violentos realizados intencional y repetitivamente por un individuo o grupo hacia una víctima con dificultades para defenderse (Smith et al., 2008), estos actos se presentan a través de las Tecnologías de la Información y la Comunicación (Buelga y Pons, 2012; Calmaestra, 2011; Kowalski, Limber y Agatston, 2008) y se dan entre niños o adolescentes que frecuentemente son compañeros de escuela (Aftab, 2010), dichos actos violentos adquieren distintas formas o tipos (Aftab, 2010; Kowalski et al., 2008).

En México, recientemente se ha prestado atención a la violencia escolar, y a la fecha se carece de datos fiables sobre su prevalencia y sus características, por lo cual se realizó una investigación en los planteles de bachillerato de la Universidad Autónoma del Estado de México cuyo objetivo fue describir la dinámica, manifestaciones y factores asociados al acoso escolar entre alumnos. Así mismo, las primeras investigaciones sobre cyberbullying aparecieron en 2009 (Del Río, Bringué, Sádaba y González; Lucio), por lo que al ser un tema que no se ha abordado ampliamente en población mexicana, se realizó un estudio cuyo objetivo fue determinar la prevalencia general de cyberbullying en alumnos de bachillerato y su prevalencia por rol de implicación y tipo.

Es importante estudiar el cyberbullying porque al sobrepasar los límites de la escuela puede presentarse a cualquier hora y en cualquier lugar, también porque las Tecnologías de la Información y la Comunicación (TIC) permiten la perdurabilidad y la de contenidos generados para acosar a la víctima y porque suele ser difícil de detectar (Kowalski et. al., 2008). También se conocen los efectos del cyberbullying para los implicados en este tipo de violencia; en el caso de las víctimas se encuentran la depresión, ansiedad, fracaso escolar, baja autoestima, estrés, falta de autoconfianza, miedo, ira, frustración, indefensión, irritabilidad, somatizaciones, dificultades de concentración, trastornos del sueño, ideación suicida y suicidio; los agresores presentan efectos como desconexión moral, carencia de empatía, incumplimiento de normas, agresividad, dificultades escolares y consumo de alcohol y drogas; los observadores también se ven afectados, disminuyendo su sensibilidad hacia las injusticas y las agresiones (Berenguí, Saldoval, De Moya y Maldonado, 2011).

Para poder tomar acciones preventivas y correctivas pertinentes, en México se realizó la Reforma Integral de la Educación Media Superior (RIEMS), (Secretaría de Educación Pública [SEP], 2008) la cual señala que los jóvenes en bachillerato deberán contar con las competencias genéricas fundamentales para que se desarrollen como personas en la sociedad y logren su comprensión del mundo, así como su influencia en él, aprendan de forma autónoma y desarrollen relaciones armónicas con quienes les rodean, así como participar eficazmente en los ámbitos social, profesional y político.

La inclusión de estas competencias genéricas en el currículum se relaciona con la recomendación de Ortega y Fernández (1998) sobre la prevención de la violencia escolar a través de un buen clima de convivencia, las autoras proponen trabajar la prevención de la violencia escolar a través del plano de la actividad, el cual incluye los contenidos curriculares que deben aprenderse en las aulas. 
Además, parte de la promoción de la convivencia para prevenir la violencia en la escuela está relacionada con la formación de la subjetividad del alumnado, que incluye sus actitudes, valores, habilidades sociales y emocionales (Ortega y Sánchez, 2004). Por lo anterior, se realizó un estudio para identificar el nivel de estimación de dominio de las competencias genéricas que los estudiantes de bachillerato de la Universidad Autónoma del Estado de México consideran que han adquirido durante su trayectoria escolar.

Respecto a los entornos virtuales, no solo hay que tener acceso a las tecnologías y no basta solamente con usarlas, sino que se debe ser competente en su uso (Cabero y Llorente, 2008); ya que el hacer un uso adecuado de las TIC y los entornos virtuales se previene el uso de estas herramientas para actos violentos. Tanta es la importancia de la competencia digital en la actualidad que la Unión Europea (Ala-Mutka, Punie y Redecker, 2008) la incluyó como una de las competencias clave, y la definió como el uso seguro y crítico de las TIC para el trabajo, el ocio y la comunicación; agregando que sus habilidades básicas incluyen el uso de ordenadores para obtener, evaluar, almacenar, producir, presentar e intercambiar información, y comunicarse y participar en redes de colaboración a través de internet.

En México se ha instrumentado el proyecto "Habilidades Digitales para Todos" (HDT) cuya aparición fue en el acuerdo 592 de la Secretaría de Educación Pública (2011), el cual sigue la línea marcada por la UNESCO (Anderson, 2010) y la Unión Europea (Ala-Mutka, et al., 2008); las habilidades que incluye el plan HDT son Creatividad e innovación, Comunicación y colaboración, Investigación y manejo de información, Pensamiento crítico, Solución de problemas y toma de decisiones y Ciudadanía digital; estás habilidades conforman en conjunto la competencia digital.

En relación con lo anterior, se obtuvieron índices de habilidades digitales en los alumnos de bachillerato de la Universidad Autónoma del Estado de México y se buscó si existía correlación con la prevalencia del cyberbullying en la población; con lo cual se buscó identificar si la competencia digital, compuesta por altos índices de habilidades digitales, podía representar un factor de protección y prevención ante el cyberbullying.

\section{MÉTODO}

\section{Estudio 1}

Se realizó un estudio descriptivo de corte transversal donde se aplicó el Cuestionario de Medición de la Agresión Escolar e intimidación en Bachillerato (CMAEIB), diseñado por Miranda, Serrano, Morales, Montes de Oca y Reynoso (2013) que tiene un Alfa de Cronbach de .920; a 12937 alumnos del bachillerato de la Universidad Autónoma del Estado de México; para describir la dinámica, manifestaciones y factores asociados al acoso escolar entre alumnos.

El CMAEIB (Mirada et al., 2013) está integrado por diez factores (actores, 
tipo de agresión, factores ecológicos, efecto de la víctima, conducta pro-social, factores de riesgo, factores ecológicos, factores contextuales, personalidad de los actores y la conflictividad), con sub-factores que en la literatura sobre el tema se plantean como variables relevantes que actúan alrededor del fenómeno de la agresión en general y del bullying en particular, teniendo en cuenta la manifestación del mismo, las características de la agresión reportadas por los estudiantes como testigo, víctima (efecto que está teniendo en ella la agresión) y/o agresor (identificación por género), así como la determinación de la frecuencia de su ocurrencia.

\section{Estudio 2}

Se realizó un estudio descriptivo transversal donde midieron las competencias genéricas de 8244 estudiantes de bachillerato de la Universidad Autónoma del Estado de México, a través de la escala estimativa de logro de competencias tipo Likert que consta de 67 ítems; las respuestas se midieron en un nivel ordinal con valores de 1 a 4, donde 1 es "no lo sé hacer", 2 es "lo hago pero necesito mejorar", 3 es "sí lo hago pero necesito mejorar" y 4 es "lo hago de manera independiente".

El instrumento considera a las competencias genéricas como aquellas que constituyen el perfil del egresado del Sistema Nacional de Bachillerato (SNB) en México; estas son las que permiten a los bachilleres comprender el mundo e influir en él, les capacitan para continuar aprendiendo de forma autónoma a lo largo de sus vidas, y para desarrollar relaciones armónicas con quienes les rodean.

Se consideran once competencias genéricas con sus respectivos atributos: Se conoce y valora a sí mismo y aborda problemas y retos teniendo en cuenta los objetivos que persigue; Es sensible al arte y participa en la apreciación e interpretación de sus expresiones en distintos géneros; Elige y practica estilos de vida saludables; Escucha, interpreta y emite mensajes pertinentes en distintos contextos mediante la utilización de medios, códigos y herramientas apropiados; Desarrolla innovaciones y propone soluciones a problemas a partir de métodos establecidos; Sustenta una postura personal sobre temas de interés y relevancia general, considerando otros puntos de vista de manera crítica y reflexiva; Aprende por iniciativa e interés propio a lo largo de la vida; Participa y colabora de 1 manera efectiva en equipos diversos; Mantiene una actitud respetuosa hacia la interculturalidad y la diversidad de creencias, valores, ideas y prácticas sociales; y Contribuye al desarrollo sustentable de manera crítica, con acciones responsables.

\section{Estudio 3}

Se utilizó un diseño no experimental de tipo transversal descriptivo y correlacional (Hernández-Sampieri, Fernández-Collado y Baptista, 2010) para medir la prevalencia de cyberbullying en alumnos de bachillerato en un único momento; 
así como para determinar la prevalencia del fenómeno en relación con la competencia digital.

Se desarrolló el instrumento "Cyberbullying y aprendizaje virtual", que fue aplicado a través de internet para determinar la prevalencia de cyberbullying tomando en cuenta las perspectivas de víctimas, agresores y observadores; los reactivos de cyberbullying para los tres implicados abarcan diez tipos basados en la clasificación sobre las conductas que se realizan. El apartado sobre aprendizaje virtual mide las habilidades digitales de Investigación y manejo de información; Creatividad e innovación; Comunicación, interacción social y colaboración; y Ciudadanía digital. Dicho instrumento obtuvo un Alfa de Cronbrach de .953, indicando una alta fiabilidad.

La población estuvo conformada por los estudiantes de los nueve planteles de bachillerato de la Universidad Autónoma del Estado de México, que en el ciclo escolar 2013-2014 registraban un total de 16127 estudiantes. Se trabajó con una muestra de 637 alumnos, se realizó un muestreo estratificado considerando turno, plantel, sexo, semestre y sexo. También se llevó a cabo un procedimiento de muestreo aleatorio simple para cada uno de los estratos que permitiera determinar que los participantes fueran elegidos al azar.

Los datos se procesaron a través del SPSS 21, donde se obtuvieron estadísticos descriptivos, y correlaciónales por medio de la correlación de Pearson.

\section{RESULTADOS}

\section{Estudio 1}

No se observa agresión en un 96.9\%, mientras que en un 3.1\% sí se presenta. Se manifiesta más agresión en los hombres que en las mujeres, siendo del $2.2 \%$ en el caso de los hombres y del .9\% en las mujeres. En cuanto al tipo de agresión reportada, el 3.1\% refirió emplear agresión física, 3.5\% de tipo sexual, 4.5\% cibernética y $4.9 \%$ daño a la propiedad ajena, destacando los porcentajes en que son reportadas de tipo verbal 7.2\% y la relacional $7.5 \%$.

En cuanto a los efectos de la agresión y el acoso, el 7.4\% de los alumnos estuvo de acuerdo o totalmente de acuerdo en que la inseguridad en la escuela es un efecto derivado de la agresión y el acoso, seguido por el ausentismo, la agresión y el aislamiento, con porcentajes mayores en los hombres, salvo en el caso de la inseguridad donde el porcentaje mayor fue de las mujeres. Del mismo modo, se les preguntó la frecuencia de ocurrencia (al menos una vez, en los últimos dos meses o diariamente) de otros efectos personales, emocionales y académicos de la agresión y el acoso. Se encontró que dos de los efectos que más impactan son el descuido personal (54.8\%) y el miedo a ser sujeto de o ver actos negativos $(49.3 \%)$, siendo en ambos casos, el porcentaje mayor el de las mujeres (30.6\% y $20.1 \%$ respectivamente); le siguen el bajo rendimiento $(13.5 \%)$, del cual no hay diferencia entre hombres y mujeres, tristeza (11.6\%) y desesperación (4.5\%), en estos dos últimos efectos destaca un mayor porcentaje en los en hombres (6.7\% y $2.8 \%$ respectivamente). 
En cuanto a los factores ecológicos, el 57\% de los alumnos considera que tiene un espacio escolar adecuado. En los factores de riesgo relacionados con adicciones y vandalismo, se encontró que el $24.5 \%$ de los jóvenes encuestados consume o sabe que consumen cigarro y alcohol entre dos a tres veces al mes y todos los días, $15.3 \%$ consume o sabe que consumen drogas y el 9.7\% realiza o sabe que realizan actos de vandalismo, el $25.6 \%$ considera que el rumbo de la escuela y su casa es peligroso e inseguro e incluso se escucha de asaltos y pandillas.

Respecto a la conflictividad entre alumno-maestro, el $11.6 \%$ de los alumnos presenta comportamientos negativos hacia los docentes, siendo estos comportamientos más altos en los hombres.

\section{Estudio 2}

La mayor parte de estudiantes $(95.1 \%)$ indica saber hacer lo que se solicita en las competencias genéricas; de ellos, solo el 37.5\% sabe hacerlo de manera independiente y $57.6 \%$ de estudiantes lo hacen pero necesitan mejorar, $4.7 \%$ de alumnos lo hacen con ayuda y el .2\% no lo saben hacer.

Respecto a las competencias genéricas, en porcentaje mayor se presenta se autodetermina y cuida de si (65\%) y en porcentaje menor trabaja en forma colaborativa (48.2\%); el 61.9\% de los estudiantes sabe escuchar, interpretar y emitir mensajes pertinentes en distintos contextos, el 60\% participa con una conciencia cívica y ética, el 59.3\% se conoce y valora a sí mismo, el 58.9\% desarrolla innovaciones y propone soluciones a problemas, el 53\% sustenta una postura personal, el 51.9\% aprende por iniciativa e interés propio, el $47.7 \%$ es sensible al arte; el 57.9\% mantiene una actitud respetuosa hacia la interculturalidad y la diversidad y el $49.3 \%$ elije y practica estilos de vida saludables de manera independiente.

Sobre las diferencias por sexo, los hombres manifiestan una estimación mayor en cuanto a saberlo hacer pero necesitar mejorar de las siguientes competencias: ser sensible al arte (49\%), escuchar, interpretar y emitir mensajes pertinentes $(61 \%)$, desarrollar innovaciones $(58 \%)$, sustentar una postura personal $(55 \%)$, aprender por iniciativa e interés propio (51\%); las mujeres manifiestan un mayor porcentaje en cuanto a saberlo hacer de manera independiente en las competencias: eligen y practican estilos de vida saludables (52\%), mantienen una actitud respetuosa (65\%) y contribuyen al desarrollo sustentable (47\%).

Acerca de la trayectoria escolar, se encontró que el nivel de dominio saberlo hacer pero necesitar mejorar disminuye de un 55\% de estudiantes en un porcentaje mínimo al $47.4 \%$ de estudiantes, cuando el semestre aumenta incrementa en relación al nivel de dominio independiente del $38.1 \%$ de estudiantes ubicados en segundo semestre al $48.7 \%$ de estudiantes en el sexto semestre.

\section{Estudio 3}

La prevalencia del cyberbullying en estudiantes de bachillerato fue del $36.1 \%$, 
con una frecuencia de incidencia variable, ya sea como víctimas, victimarios $\mathrm{u}$ observadores y tomando en cuenta todos los tipos abordados de cyberbullying. De forma particular, la prevalencia de víctimas de cyberbullying fue del $23.86 \%$, la de victimarios fue del 7.69\% y la de observadores del 67.66\%. En la Tabla 1 se presentan las medias y desviaciones estándares que se presentaron por roles.

Tabla 1. Media y Desviaciones Típicas de las Variables del Estudio 3

\begin{tabular}{lcc}
\hline \multicolumn{1}{c}{ Variable } & Media & Desviación Típica \\
\hline Investigación y manejo de información & 2.08 & .925 \\
Creatividad e innovación & 2.21 & 1.040 \\
Comunicación, interacción social y & 2.00 & .967 \\
colaboración & & .986 \\
Cuidadanía Digital & 2.40 & .468 \\
Cyberbullying (Victima) & .25 & .321 \\
Cyberbullying (Victimario) & .08 & \\
\hline
\end{tabular}

La prevalencia de cyberbullying por tipos de conducta que se realiza se obtuvo de forma total para cada uno, considerando a los tres roles implicados. La prevalencia de insultos electrónicos fue del 77.23\%, la de hostigamiento fue del $42.22 \%$, la denigración mostró una prevalencia de $38.77 \%$, la suplantación obtuvo un $48.82 \%$, desvelamiento y sonsacamiento mostraron una prevalencia de $26.37 \%$, las actividades relacionadas con exclusión y ostracismo obtuvieron un porcentaje del 59.96, la ciberpersecución obtuvo una prevalencia del $26.06 \%$, la paliza feliz obtuvo un porcentaje del 26.53 , se detectó una prevalencia de $21.50 \%$ de sexting y otras formas de violencia (que solo incluye contenido maltrato a otros) mostró una porcentaje del 30.92 .

En relación a las habilidades digitales que conforman la competencia digital, las medias y desviaciones típicas encontradas en la muestra del estudio se muestran en la Tabla 1, siendo estas cercanas al 2, lo cual indica que todas estas competencias son utilizadas solo algunas veces en los entornos virtuales por los sujetos de la muestra.

En cuanto a cómo se relacionan estas competencias digitales con los roles de víctima, victimario y observador, las correlaciones estadísticamente signifi- 
cativas al nivel de .01 encontradas son las siguientes: Investigación y manejo de información con los roles de víctima $(r=.112)$, victimario $(r=130) \mathrm{y} \mathrm{ob-}$ servador $(r=.198)$; Creatividad e innovación con los roles de víctima $(r=.117)$, victimario $(r=.122)$ y observador $(r=.272)$; Comunicación, interacción social y colaboración con los roles de víctima $(r=.133)$, victimario $(r=.171)$ y observador $(r=.236)$; y Ciudadanía digital solo correlacionó con el rol de observador $(r=.169)$.

\section{CONCLUSIONES}

Como se mostró en los resultados, la prevalencia de bullying (3.1\%) fue más baja que la de cyberbullying (36.1\%) en la población estudiada, siendo oportuna la prevención primaria y secundaria, así como la intervención sobre estas problemáticas para que no se incrementen e incluso para disminuirlas.

Hay que resaltar que la prevalencia del $36.1 \%$ de cyberbullying en estudiantes de bachillerato puede parecer alta debido a que incluye niveles de frecuencia variable, y dentro de estos niveles, la mayoría de los casos se han presentado casi nunca. El porcentaje de prevalencia general también incluye a los tres roles de implicados, así como los tipos de conducta que se realizan. Por separado, la prevalencia de víctimas fue del $23.86 \%$, la de victimarios del $7.69 \%$ y la de observadores del $67.66 \%$.

Por otra parte, las competencias genéricas son aquellas enfocadas al desempeño ciudadano que incluyen aspectos de ciudadanía y convivencia; se consideran atributos generales de los alumnos que pueden ser manifestadas en sus diferentes desempeños en diferentes contextos (Victorino y Medina, 2011), por lo cual se dice que estas son clave, transversales y transferibles (SEP, 2008). En general, se espera que con la implementación de las competencias genéricas en el currículum a partir de la Reforma Integral del Nivel Medio Superior en el nivel bachillerato mexicano, los estudiantes alcancen un desarrollo personal sano y relaciones sociales armónicas con los otros.

En general, se encontraron índices altos de competencias genéricas, los porcentajes más elevados fueron los correspondientes a las competencias de se autodetermina y cuida de sí (65\%), saber escuchar, interpretar y emitir mensajes pertinentes en distintos contextos $(61.9 \%)$ y participar con una conciencia cívica y ética (60\%). Estas competencias se consideran conductas incompatibles con la violencia escolar en general y el bullying en particular, por lo que su inclusión curricular puede representar una medida de prevención ante estas problemáticas (Miranda, Serrano, Morales y Delgado, 2012). Krauskopf (2006) apoya dicha noción identificando que el desarrollo de competencias y habilidades para la convivencia social es una medida de prevención de la violencia escolar.

De la misma forma, se han desarrollado algunos estudios para la prevención e intervención de la violencia escolar y el bullying a través de competencias y habilidades generales o específicas. En la línea del desarrollo de competencias genéricas incompatibles con la presencia de violencia escolar, se encuentran tra- 
bajos como los de Cortes (2009) y Jiménez-Hernández (2012). Otros trabajos se han enfocado en competencias y habilidades específicas, principalmente en el desarrollo de competencias socioemocionales, sociales y emocionales (Extremera y Fernández-Berrocal, 2004; Ortega, 2007; Repetto y Pena, 2010; Repetto, Pena, Mudarra y Uribarri, 2007; Sánchez, Ortega y Menesini, 2012).

Considerando los trabajos consultados y la presencia de índices altos de competencias genéricas acompañados por baja prevalencia de bullying, el fortalecimiento de las competencias genéricas parece una medida de prevención de la violencia escolar a través del cumplimiento de las metas del currículum que son incompatibles con estos fenómenos.

Sin embargo, al referirnos a la prevención de bullying a través de las competencias genéricas existe la limitante de que estas temáticas se abordaron en dos estudios separados; pero al tratarse de la misma población, se establece la relación de bajos índices de bullying y un buen dominio de competencias genéricas de los alumnos; por lo anterior, podemos deducir que los estudiantes poseen las herramientas para saber cómo reaccionar ante situaciones de bullying utilizando las competencias genéricas.

En el caso del cyberbullying y la competencia digital, en el estudio 3 se encontró que en relación con las habilidades digitales, el rol de víctima presenta menor índice de correlación, el de agresor manifiesta mediano índice y el de observador presenta el índice más elevado; indicando que a un mayor grado de habilidades digitales es más probable que los jóvenes solo desempeñen el rol de observador. En el caso de la ciudadanía digital, esta solo tuvo correlación con el rol de observador debido a que esa habilidad implica el uso respetuoso y ético de los entornos virtuales y las TIC, indicando que al desarrollar esta habilidad se pueden prevenir los actos de cyberbullying.

Por lo tanto, se plantea que la prevención de cyberbullying puede realizarse por medio del desarrollo de la competencia digital, ya sea incluyéndola en el currículum o a través de un programa que trabaje la instrucción y concientización de los alumnos en lo referente las habilidades digitales, particularmente a través de la ciudadanía digital.

\section{REFERENCIAS}

Aftab, P. (2010). What is cyberbullying, exactly? Recuperado el 16 de octubre de 2014 de http://stopcyberbullying.org/what is_cyberbullying_exactly.html Ala-Mutka, K., Punie, Y. y Redecker, C. (2008). Digital competence for lifelong Learning. European Commission, Institute for Prospective Technological Studies. Recuperado el 23 de octubre de 2014 de http://ftp.jrc.es/EURdoc/ JRC48708.TN.pdf

Anderson, J. (2010). ICT TransformingEducation. A Regional Guide. Organización de las Naciones Unidas para la Educación, la Ciencia y la Cultura (UNESCO). Recuperado el 02 de noviembre de 2014 de http://unesdoc. unesco.org/images/0018/001892/189216E.pdf 
Berenguí, A., Saldoval, C., De Moya, J. y Maldonado, I. (2011). Plan de intervención: Cyberbullying. Recuperado el 20 de octubre de 2014 de http://issuu. com/irenemaldonado/docs/plan_de_intervencion_innovacion

Buelga, S. y Pons, J. (2012). Agresiones entre Adolescentes a través del Teléfono Móvil y de Internet. Psychosocial Intervention 21(1), 91-101. Recuperado 20 de septiembre de 2014 de http://www.redalyc.org/articuloBasic. oa? id $=179824383008$

Cabero, J. y Llorente M. (2008). La alfabetización digital de los alumnos. Competencias Digitales para el siglo XXI. Revista portuguesa de Pedagogía. Núm. 42. P. 7-28. Recuperado 29 de septiembre de 2014 de http://iduc.uc.pt/ index.php/rppedagogia/article/viewFile/1234/682.

Calmaestra, J. (2011). Cyberbullying: Prevalencia y Características de un nuevo tipo de bullying indirecto. Tesis doctoral. Servicio de Publicaciones de la Universidad de Córdoba. Recuperado el 20 de octubre del 2014 de http://helvia.uco.es/xmlui/bitstream/handle/10396/5717/9788469490976. pdf? sequence $=1$

Cortes, M. A. (2009). Aprendizaje cooperativo como herramienta para eliminar el bullying en los centros educativos. Innovación y experiencias educativas, 45. Recuperado el 3 de febrero de 2015 de http://www.csi-csif.es/andalucia/ modules/mod_ense/revista/pdf/Numero_25/MARIA_CRISTINA_CORTES_ARIZA02.pdf

Del Rey, R. y Ortega, R. (2007). Violencia escolar: claves para comprenderla y afrontarla. Escuela Abierta, 10, 77-89. Recuperado el 4 de febrero de 2015 de http://www.researchgate.net/profile/Ortega_Rosario/publication/28203713_Violencia_escolar_claves_para_comprenderla_y_afrontarla/ links/0912f50b6914cbf02e000000.pdf

Del Río, J., Bringué, X., Sádaba, C. y González, D. (2009). Cyberbullying: un análisis comparativo en estudiantes de Argentina, Brasil, Chile, Colombia, México, Perú y Venezuela. Recuperado el 6 de septiembre de 2014 de http:// www.rua.unam.mx/objeto/8836/cyberbullying-un-analisis-comparativo-enestudiantes-de-argentina-brasil-chile-colombia-mexico-peru-y-venezuela

Extremera, N. y Fernández-Berrocal, P. (2004). El papel de la inteligencia emocional en el alumnado: evidencias empíricas. Revista Electrónica de Investigación Educativa, 6 (2). Recuperado el 13 de diciembre de 2014 de: http:// redie.uabc.mx/vol6no2/contenido-extremera.html

Hernández-Sampieri, R., Fernández-Collado, C. y Baptista-Lucio, P. (2010). Metodología de la investigación (5ta ed.). México: Mc Graw Hill.

Jiménez-Hernández, J. C. (2012). El Bullying y las competencias ciudadanas. Recuperado el 28 de diciembre de 2014 de http://www.colegionicolasesguerra.edu.co/images/documentos/2Informe-de-investigacion-Bullyng.pdf

Kowalski, R., Limber, S. y Agatston, P. (2008). Cyber Bullying: El acoso escolar en la era digital. Urduliz: Desclée De Brouwer.

Krauskopf, D. (2006). Estado del arte de los programas de prevención de la violencia en ámbitos escolares. Recuperado el 17 de enero de 2015 de http:// 
www.paho.org/hq/dmdocuments/2009/arte.violencia.escolares.pdf?ua=1

Lucio, L.A. (2009). Agresores escolares en el ciberespacio; el cyberbullying en preparatorias mexicanas. Trabajo presentando en XI Asamblea General de ALAFEC. Guayaquil-Ecuador, 22 al 25 de septiembre (paper).

Miranda, D. A., Serrano, J. M., Morales, T. y Delgado, M. E. (2012). Diagnóstico de la agresión e intimidación en los planteles de la Escuela Preparatoria de la UAEMéx. Recuperado el 23 de septiembre de 2014 de https://www.google. com.mx/url sa $=$ t\&rct $=\mathrm{j} \& \mathrm{q}=\&$ esrc $=\mathrm{s} \&$ source $=$ web\&cd $=1 \& \mathrm{cad}=\mathrm{rja} \& u a c t=8$ \&ved=0CB0QFjAA\&url=http $\% 3 \mathrm{~A} \% 2 \mathrm{~F} \% 2 \mathrm{Fwww}$. ipomex.org.mx $\% 2 \mathrm{Fipo} \%$ 2Farchivos\%2FdownloadAttach\%2F255035.web\%3Bjsessionid\%3DB5244 94A78F203A85BCDC98B3E8F1EB2\&ei=gIGEVd3BCsOvsAW_gYKoDA \&usg=AFQjCNG21XVGN7880Hbyiw5Qgg9fIFclbQ\&sig2=hxmrUP4vHb T4FzPIUy33kg\&bvm=bv.96339352,d.b2w

Miranda, D. A., Serrano, J. M., Morales, T., Montes de Oca, J. Z. y Reynoso, B. (2013). "Agresión y acoso en el nivel medio superior: el caso de la Universidad Autónoma del Estado de México". En Pedroza, F. J. y Rubalcava, S. J. (Coord.). La Construcción de identidades agresoras: el acoso escolar en México. México: Consejo Nacional para la Cultura y las Artes (CONACULTA).

Olweus, D. (2006). Conductas de acoso y amenaza entre escolares. Lima: Alfaomega.

Ortega, R. (2007). Competencias para la convivencia y las relaciones sociales. Cuadernos de pedagogía, 370, 32-35. Recuperado el 13 de septiembre de 2014 de http://www.documentacion.edex.es/docs/0401 pozcom.pdf

Ortega, R. y Fernández, F. (1998). Un proyecto educativo para mejorar la convivencia y prevenir la violencia. En Ortega, R. La Convivencia Escolar: qué es y cómo abordarla (pp. 79-94). Recuperado el 16 de febrero de 2014 de http:// harcelement-entre-eleves.com/images/presse/convivenciaqosarioortega.pdf

Ortega, R. y Sáchez, V. (2004). Construir la convivencia para prevenir la violencia. Aula de innovación educativa, 132, 70-74. Recuperado el 16 de febrero de 2014 de: http://213.0.8.18/portal/Educantabria/RECURSOS/Proyecto/SopoDOC/01_Construir.pdf

Repetto, E. y Pena, M. (2010). Las competencias socioemocionales como factor de calidad en la educación. Revista Iberoamericana sobre Calidad, Eficacia y Cambio, 8(5). Recuperado el 23 de diciembre de 2014 de http://www.rinace. net/reice/numeros/arts/vol8num5/art5 htm.htm

Repetto, E., Pena, M., Mudarra, M. J. y Uribarri, M. (2007). Orientación de las competencias socioemocionales de los alumnos de Educación Secundaria en contextos multiculturales. Revista Electrónica de Investigación Psicoeducativa, 11 (5), 159-178. Recuperado el 13 de noviembre de 2014 de http://www. investigacion-psicopedagogica.org/revista/new/ContadorArticulo.php?170

Sánchez, V., Ortega, R. y Menesini, E. (2012). La competencia emocional de agresores y víctimas de bullying. Anales de Psicología, 28(1), 71-82. Recuperado el 15 de diciembre de 2014 de http://www.redalyc.org/pdf/167/16723161009. pdf 